\title{
Synthesis of acylhydrazino-peptomers, a new class of peptidomimetics, by consecutive Ugi and hydrazino-Ugi reactions
}

\author{
Angélica de Fátima S. Barreto*, Veronica Alves dos Santos \\ and Carlos Kleber Z. Andrade*
}

\section{Full Research Paper}

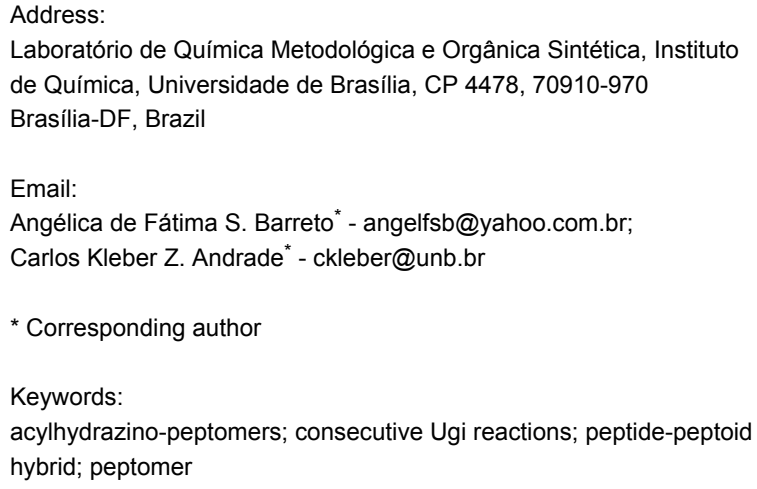

Laboratório de Química Metodológica e Orgânica Sintética, Instituto de Química, Universidade de Brasília, CP 4478, 70910-970 Brasília-DF, Brazil

Email:

Angélica de Fátima S. Barreto* - angelfsb@yahoo.com.br;

Carlos Kleber Z. Andrade* - ckleber@unb.br

* Corresponding author

Keywords:

acylhydrazino-peptomers; consecutive Ugi reactions; peptide-peptoid

hybrid; peptomer

\author{
Beilstein J. Org. Chem. 2016, 12, 2865-2872. \\ doi:10.3762/bjoc. 12.285 \\ Received: 28 September 2016 \\ Accepted: 21 December 2016 \\ Published: 27 December 2016 \\ Associate Editor: N. Sewald \\ (c) 2016 Barreto et al.; licensee Beilstein-Institut. \\ License and terms: see end of document.
}

\begin{abstract}
Herein we describe a versatile approach for the synthesis of acylhydrazino-peptomers, a new class of peptidomimetics. The key idea in this approach is based on a simple route using a one-pot hydrazino-Ugi four-component reaction followed by a hydrazinolysis or hydrolysis reaction and subsequent hydrazino-Ugi reaction or classical Ugi reaction for the construction of acyclic acylhydrazino-peptomers. The consecutive multicomponent reactions produced a variety of acylhydrazino-peptomers in moderate to excellent yields (47-90\%). These compounds are multifunctional intermediates that can be further functionalized to obtain new peptidomimetics with potential biological activity.
\end{abstract}

\section{Introduction}

In the last decades, increasing efforts have been extensively carried out to improve the pharmacological properties of natural peptides by structural modification of the amino acids [1-8]. These modifications allowed the obtention of molecules that mimic the properties of peptides (peptidomimetics) but usually exhibit greater proteolytic stability, increased cellular permeabilities and avoid stereochemical constraints. Figure 1 represents some of the most important classes of peptidomimetics so far obtained and highlights the differences among them. Of these, peptoids (oligomers of $N$-substituted glycine residues) [9-12] are the most common and may have interesting biological activities. For instance, peptoid 1 is a target for cancer therapeutics for being an antagonist of the vascular endothelial growth factor receptor 2 [13]; peptoid 2 is a ligand of the protooncogene Crk [14]; and peptoids 3 and $\mathbf{4}$ showed a high affinity for the $\alpha 1$-adrenergic and $\mu$-specific opiate receptors [15], respectively (Figure 2). 


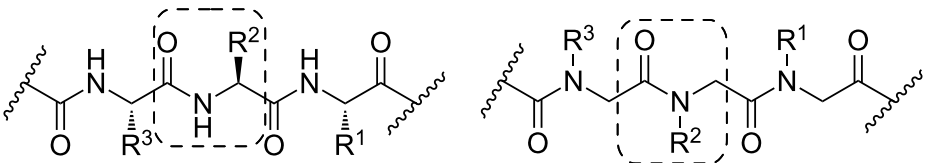

(a) peptide

(b) peptoid

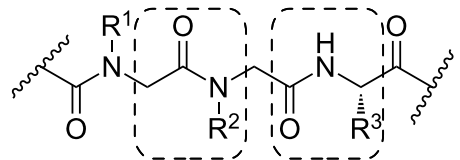

(c) peptomer

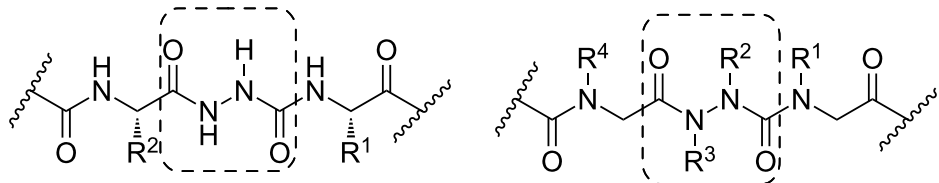

(d) azapeptide

(e) azapeptoid

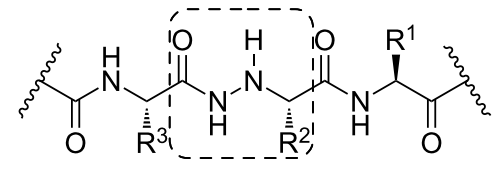

(f) hydrazinopeptide

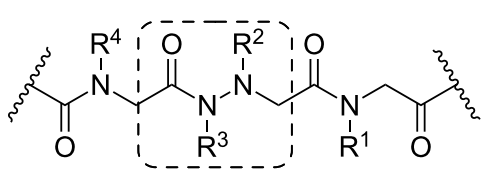

(g) hydrazinopeptoid

Figure 1: Structural features of (a) peptide, (b) peptoid, (c) peptomer, (d) azapeptide, (e) azapeptoid, (f) hydrazinopeptide and (g) hydrazinopeptoid<smiles>CC(C)CN(CC(=O)N(CCCCN)CC(=O)N(CC(=O)N(CCCCN)CC(=O)N(CCCCN)CC(=O)N(CC(=O)N(CCCCN)CC(N)=O)CC(C)C)Cc1ccc2c(c1)OCO2)C(=O)CNCCCCN</smiles><smiles>C[C@H](c1ccccc1)N(CC(N)=O)C(=O)CN(Cc1ccccc1)C(=O)CN(C(=O)CN(CCO)C(=O)CN(C(=O)CN(CCO)C(=O)CN(CCCCN)C(=O)CNCCN(CCN)CCN)C1CC1)C1CCC(O)CC1</smiles><smiles>NC(=O)CN(CCc1ccccc1)C(=O)CN(C(=O)CNCCc1ccc(O)cc1)c1ccc(-c2ccccc2)cc1</smiles><smiles>NC(=O)CN(CCc1ccc(O)cc1)C(=O)CN(Cc1ccc2c(c1)OCO2)C(=O)CNCC(c1ccccc1)c1ccccc1</smiles>

Figure 2: Some biologically active peptoids. 
Unlike peptides, in peptoids the side chain of the $\mathrm{C}^{\alpha}$ is bound to the nitrogen atom. Due to the consequent lack of the polar $\mathrm{N}-\mathrm{H}$ bonds, their lipophilicity is increased, which may result in improved membrane permeability [16,17]. Furthermore, peptoids have also found utility in supra- and macromolecular engineering [18] and polymer chemistry [19]. Nevertheless, the lack of constraints in peptoids may result in a lower affinity for macromolecular targets, which limits their utility [20]. This finding has motivated the search for efficient strategies to introduce structural constraints into peptoid structures, which has contributed to increase the plethora of currently available peptidomimetics.

Azapeptides [21-23] are peptide analogs in which the $\mathrm{C}^{\alpha}$ atom is substituted by a nitrogen atom in one or more amino acids (Figure 1) and have been known since 1970 [24]. The introduction of a semicarbazide moiety has an enormous effect on the physical properties of a peptide as well as on its structural characteristics. The semicarbazide constraints tend to facilitate interactions with protein receptors due to turn geometry within the azapeptide. As a result, their stability over enzymes and chemical degradation may be enhanced compared to a natural peptide. Indeed, these compounds have shown to be a useful class of peptidomimetics with interesting biological activities [21-23], including antiviral $[25,26]$ and cysteine protease inhibition [2730].

Hydrazinopeptides [31-36] (peptide analogs in which one of the CONH links is replaced by a hydrazido fragment CONHNH, Figure 1) represent another class of peptidomimetics with promising conformational and biological activities, such as protease inhibition [37] and antimicrobial activity [38]. There are some natural peptides that contain such an $\alpha$-hydrazino acid moiety, e.g., the vitamin B6 antagonist linatine and the antibiotic negamycin (Figure 3). In the early 1970s, the first attempts to peptide modifications by hydrazino acids generated bioactive pseudopeptides [39]. Analogously to azapeptides, these compounds possess a conformational constraint (hydrazino turn) due to the presence of intramolecular H-bonding interactions (Figure 3), which is similar in nature to a natural peptide $\beta$-turn. As already pointed out, this can improve the proteolytic stability of the natural peptide while preserving its biological activity.

Other changes in peptoid structures have also been carried out to obtain the less common but also biologically active azapeptoids [40], hydrazino-azapeptoids [41,42], retro hydrazinoazapeptoids [43] and peptoid-azapeptoid hybrids [39]. Recently, Seo et al. [44] reported the synthesis of a library of peptidepeptoid hybrid (termed peptomers by Ostergaard and Holm [45]) prodrugs that can be selectively activated by prostate cancer cells.

Peptidomimetics can be conveniently synthesized using the so called "submonomer approach" either in solution [41] or in solid-phase [46,47]. However, some disadvantages have been reported for long or difficult sequences [48]. It is therefore important to have alternative methods for the fast and easy construction of such important compounds. In this sense, the Ugi four-component reaction (U-4CR) has proven to be a robust and versatile method for the synthesis of peptoids and peptidepeptoid hybrids (peptomers) [49-53]. This reaction has also been combined with other protocols for the synthesis of bioactive peptides [54,55] and hydrazinopeptide motifs [33,34]. In continuing our research on the synthesis of peptoids [53,56,57], herein we describe the synthesis of a new class of peptidomimetics, which we have called acylhydrazino-peptomers (Figure 4), by analogy with the existing classes of peptidomimetics shown in Figure 1, using consecutive Ugi and hydrazino-Ugi reactions, respectively. These compounds comprise both a peptoid and a peptide moiety (hence a peptomer) along with an acylhydrazino portion. The use of a consecutive Ugi reaction to access peptidomimetics has proven very useful [56-62]. It is important to point out that these molecules cannot be obtained directly via the "submonomer approach".

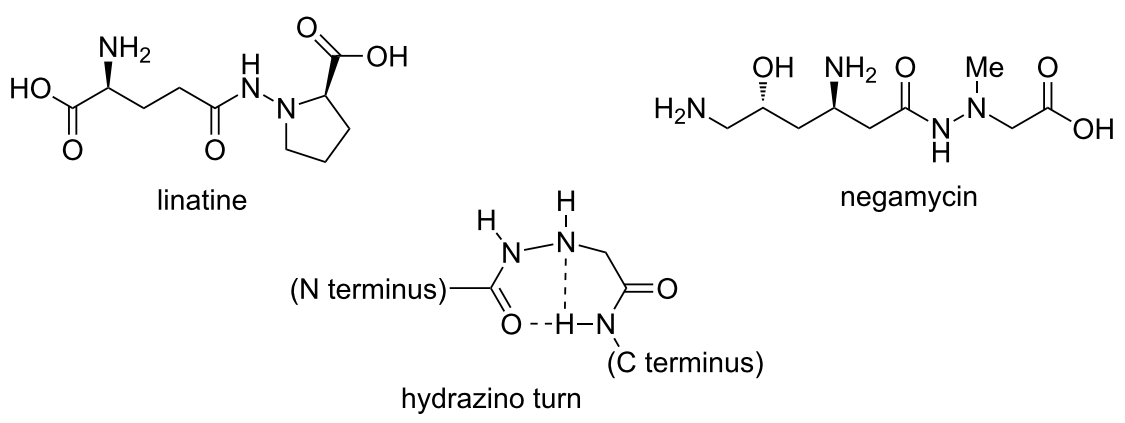

Figure 3: Biologically active hydrazinopeptides and representation of the hydrazino turn. 


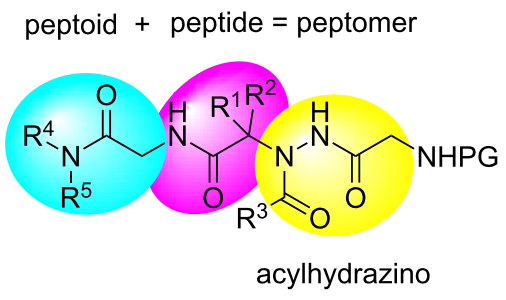

Figure 4: General structure of the acylhydrazino-peptomers synthesized in this study.

\section{Results and Discussion}

Our approach involves the use of two multicomponent reactions (Scheme 1): the hydrazino-Ugi four-component reaction (HU-4CR) and the classical Ugi reaction (U-4CR). The strategy was based on the formation of an acylhydrazino-peptomer via an initial hydrazino-Ugi reaction followed by a hydrazinolysis reaction (or ester hydrolysis) and a subsequent hydrazino-Ugi reaction (or a classical Ugi reaction).

The hydrazides 3a-c used in the first MCR were prepared by the reaction of glycine-derived esters $\mathbf{2 , 5}$ and $\mathbf{7}$ with hydrazine monohydrate (hydrazinolysis), following a known procedure [63,64] (Scheme 2).

The obtained hydrazides were then reacted with isobutyraldehyde $\mathbf{8 a}$ /acetone $\mathbf{8 b}$, carboxylic acids $\mathbf{1 0 a}-\mathbf{c}$ (formic, acetic or propionic acid) and ethyl isocyanoacetate 9 (Scheme 3 ). The reactions were conducted at room temperature in trifluoroethanol (TFE) for 1-2 days to yield the acylhydrazinopeptomers 11a-f in moderate to good yields (59-90\%). Aliphatic aldehydes, ketones and carboxylic acids succeeded in the hydrazino-Ugi reaction, except for paraformaldehyde, fatty acids and aromatic substrates, which led to formation of a com-

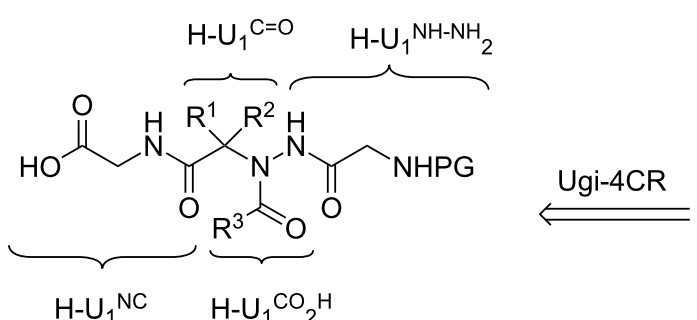

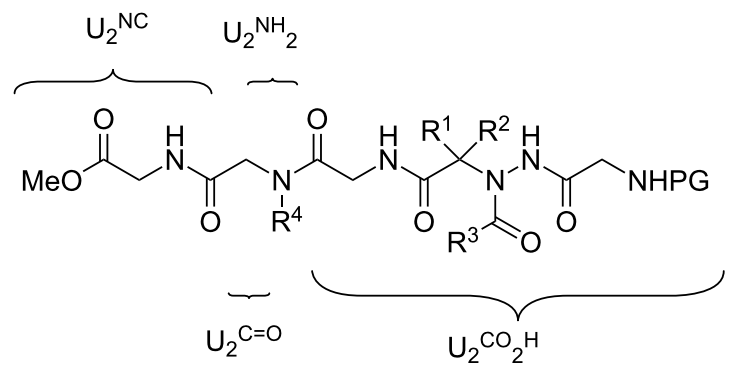
hydrazino-Ugi-4CR<smiles>[R5]NCC(=O)N[CH-][NH2+]CC(=O)OC([R])=O</smiles>
hydrazino-Ugi-4CR

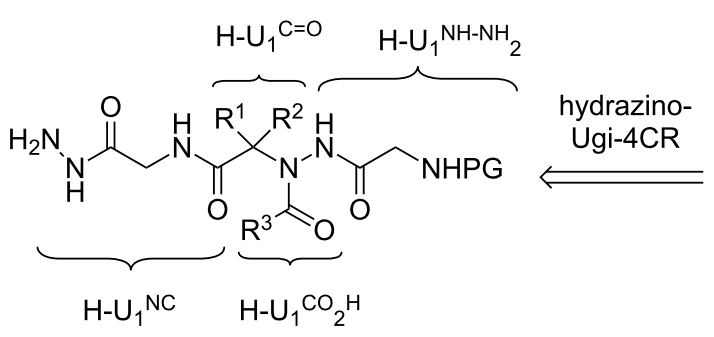

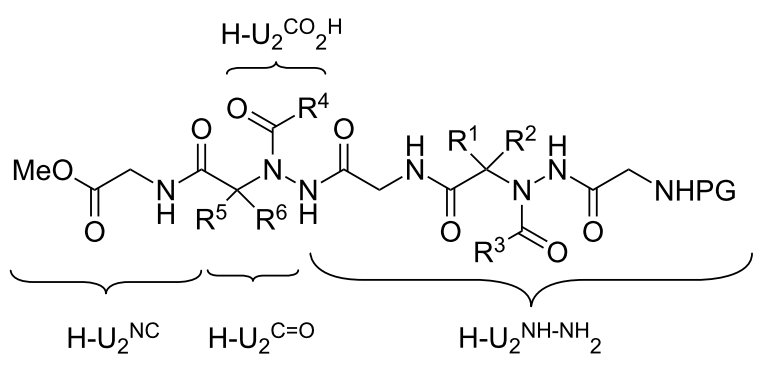



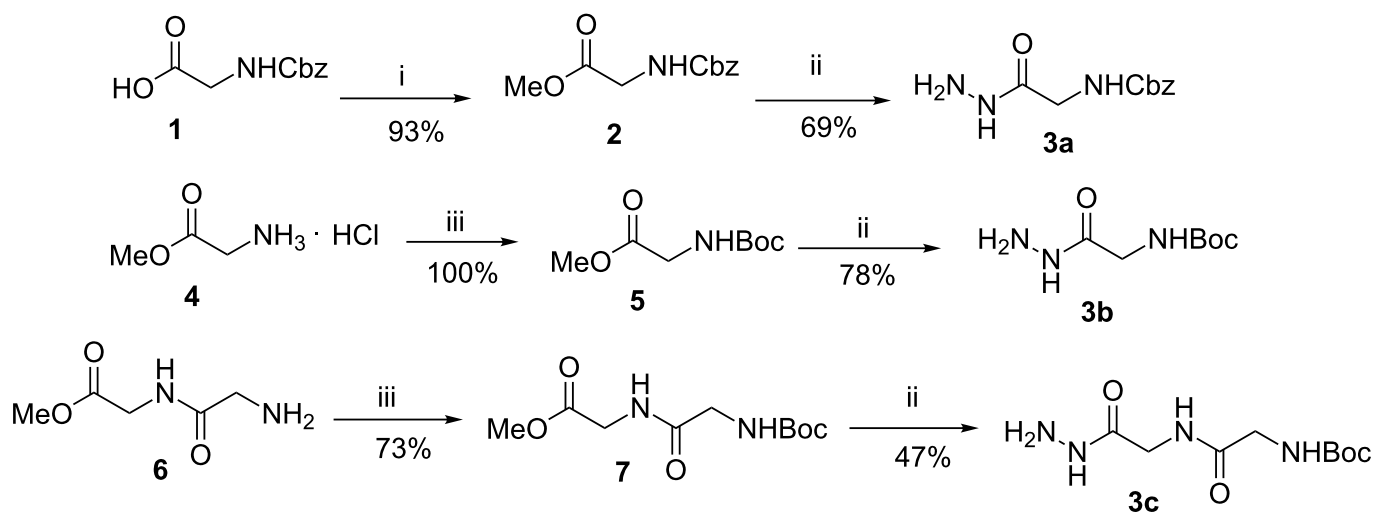

Scheme 2: Synthesis of hydrazides 3a-c. Reagents and conditions: (i) $\mathrm{CH}_{3} \mathrm{l}, \mathrm{NaHCO}_{3}$, DMF, rt, $46 \mathrm{~h}$; (ii) $\mathrm{N}_{2} \mathrm{H}_{4} \cdot \mathrm{H} \mathrm{H}_{2} \mathrm{O}, \mathrm{EtOH}$, reflux, 2-3 h; (iii) (Boc) ${ }_{2} \mathrm{O}$, $\mathrm{NaOH}$, dioxane $/ \mathrm{H}_{2} \mathrm{O}$, overnight.

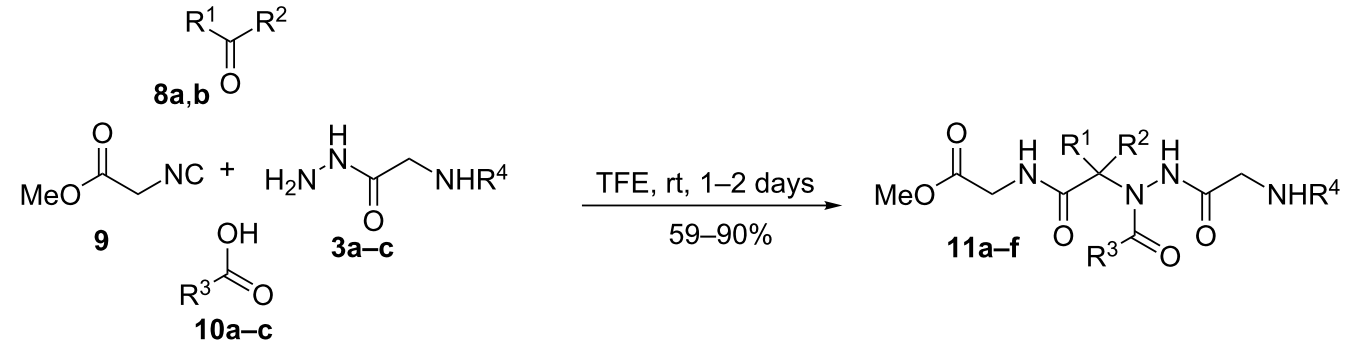
3a: $R^{4}=C b z$
3b: $R^{4}=$ Boc
3c: $\mathrm{R}^{4}=\mathrm{COCH}_{2} \mathrm{NHBOC}$
8a: $\mathrm{R}^{1}=\mathrm{H}, \mathrm{R}^{2}=\mathrm{CH}\left(\mathrm{CH}_{3}\right)_{2}$
8b: $\mathrm{R}^{1}=\mathrm{CH}_{3}, \mathrm{R}^{2}=\mathrm{CH}_{3}$
10a: $R^{3}=H$
10b: $\mathrm{R}^{3}=\mathrm{CH}_{3}$
10c: $\mathrm{R}^{3}=\mathrm{CH}_{2} \mathrm{CH}_{3}$<smiles>COC(=O)CNC(=O)C(C(C)C)N(NC(=O)CNC(C)=O)C(C)=O</smiles><smiles>COC(=O)CNC(=O)C(C(C)C)N(NC(=O)CNC(C)=O)C(C)=O</smiles>

11a, $69 \%$

$11 b, 59 \%$<smiles>CCCCCCCC(C)(C)OC(=O)NCC(=O)NN(C(=O)CNC(=O)OC)C(C)(C)C(=O)OC</smiles><smiles>COC(=O)CNC(=O)C(C)(C)N(NC(=O)CNC(=O)OCc1ccccc1)C(=O)OC(C)(C)C</smiles><smiles>COC(=O)CNC(=O)C(C(C)C)N(NC(=O)CNC(=O)CNC(=O)OCc1ccccc1)C(C)=O</smiles>

11 e, $73 \%$<smiles>CCC(=O)N(NC(=O)CNC(=O)CNC(=O)OCc1ccccc1)C(C(=O)NCC(=O)OC)C(C)C</smiles>

Scheme 3: Synthesis of acylhydrazino-peptomers 11a-f.

plex mixture. These results limited the obtention of a greater variety of acylhydrazino-peptomers by this method. The choice of TFE as the solvent for the hydrazino-Ugi reaction was important because the same reaction carried out in methanol, often the solvent of choice for Ugi reactions, provided the formation of a complex mixture. This fact has already been reported [34].
To further functionalize the acylhydrazino-peptomers structures, a second Ugi reaction was carried out. Hence, some acylhydrazino-peptomers were subjected to hydrazinolysis reaction or ester hydrolysis to give the corresponding hydrazides 12a,b (Scheme 4) or acids 13a-c (Scheme 5), respectively, which were used in the following step (hydrazino-Ugi reaction or clas- 


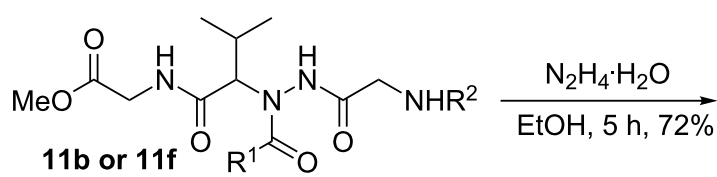

$\mathrm{R}^{1}=\mathrm{CH}_{3}, \mathrm{R}^{2}=\mathrm{Boc}$

$\mathrm{R}^{1}=\mathrm{CH}_{2} \mathrm{CH}_{3}, \mathrm{R}^{2}=\mathrm{COCH}_{2} \mathrm{NHBoC}$<smiles>[R]NCC(=O)NN(C([R])=O)C(C(=O)NCC(=O)NN)C(C)C</smiles><smiles>CCCC(=O)OCC(C)=O</smiles>

$8 b$<smiles>[R]NCC(=O)NN(C([R7])=O)C(C(=O)NCC(=O)NN(C(=O)CC)C(C)(C)C(=O)NCC(=O)OC)C(C)C</smiles>

14a, $\mathrm{R}^{1}=\mathrm{CH}_{3}, \mathrm{R}^{2}=\mathrm{Boc}(60 \%)$

14b, $\mathrm{R}^{1}=\mathrm{CH}_{2} \mathrm{CH}_{3}, \mathrm{R}^{2}=\mathrm{COCH}_{2} \mathrm{NHBoc}(90 \%)$

Scheme 4: Synthesis of acylhydrazino-peptomers 14a,b.<smiles>[R1]NCC(=O)NN(C([R])=O)C([R])([R])C(=O)NCC(=O)OC</smiles>

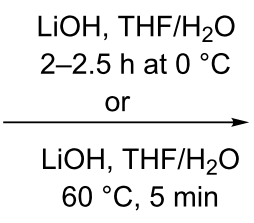<smiles>[R1]NCC(=O)NN(C([R])=O)C([R])([R])C(=O)NCC(=O)O</smiles>

$\mathrm{MeOH}, 24 \mathrm{~h}, \mathrm{rt}$

or

$80^{\circ} \mathrm{C}, 3 \mathrm{~min}$

$\mathrm{MeOH}, \mathrm{MW}$<smiles>COC(=O)CCOCCNc1ccccc1</smiles><smiles>[R]NCC(=O)NN(C([R])=O)C([R])([R])C(=O)NCC(=O)N(CC(=O)NCC(=O)OC)Cc1ccccc1</smiles>

15a $\mathrm{R}^{1}=\mathrm{H}, \mathrm{R}^{2}=\mathrm{CH}\left(\mathrm{CH}_{3}\right)_{2}, \mathrm{R}^{3}=\mathrm{CH}_{3}, \mathrm{R}^{4}=\mathrm{Cbz}(47 \%)$

15b R $\mathrm{R}^{1}=\mathrm{H}, \mathrm{R}^{2}=\mathrm{CH}\left(\mathrm{CH}_{3}\right)_{2}, \mathrm{R}^{3}=\mathrm{CH}_{3}, \mathrm{R}^{4}=\mathrm{Boc}(76 \%)$

15c R $\mathrm{R}^{1}=\mathrm{CH}_{3}, \mathrm{R}^{2}=\mathrm{CH}_{3}, \mathrm{R}^{3}=\mathrm{CH}_{2} \mathrm{CH}_{3}, \mathrm{R}^{4}=\operatorname{Boc}(55 \%)$ sical Ugi reaction) to yield the corresponding acylhydrazinopeptomers 14a,b or 15a-c in moderate to good yields (47-90\%). All compounds were fully characterized by ${ }^{1} \mathrm{H}$ and ${ }^{13} \mathrm{C}$ NMR and HRMS giving data consistent with the proposed structures.

Functionalization on both termini of compounds 11, 14 and 15 (ester hydrolysis and/or Boc deprotection) allows subsequent
Ugi or hydrazino-Ugi reactions to further elongate the peptomers main chain.

\section{Conclusion}

In summary, we have developed a concise protocol for the synthesis of functionalized acylhydrazino-peptomers by consecutive Ugi reactions. The general route allows an easy access to highly functionalized peptomers in good yields and a reduced 
number of steps. This method may also be employed to obtain new classes of peptidomimetics with potential biological activity.

\section{Supporting Information}

\section{Supporting Information File 1}

Detailed experimental procedures, NMR and mass spectra of all compounds.

[http://www.beilstein-journals.org/bjoc/content/ supplementary/1860-5397-12-285-S1.pdf]

\section{Acknowledgements}

The authors thank the Instituto de Química, Universidade de Brasília, CAPES and CNPq for financial support.

\section{References}

1. Zuckermann, R. N.; Kodadek, T. Curr. Opin. Mol. Ther. 2009, 11, 299-307.

2. Simpson, L. S.; Burdine, L.; Dutta, A. K.; Feranchak, A. P.; Kodadek, T. J. Am. Chem. Soc. 2009, 131, 5760-5762. doi:10.1021/ja900852k

3. Mora, P.; Masip, I.; Cortés, N.; Marquina, R.; Merino, R.; Merino, J.; Carbonell, T.; Mingarro, I.; Messeguer, A.; Pérez-Payá, E. J. Med. Chem. 2005, 48, 1265-1268. doi:10.1021/jm040834i

4. Fowler, S. A.; Stacy, D. M.; Blackwell, H. E. Org. Lett. 2008, 10, 2329-2332. doi:10.1021/ol800908h

5. Wenger, R. M.; Payne, T. Prog. Clin. Biol. Res. 1989, 291, 301-305.

6. Hamy, F.; Felder, E. R.; Heizmann, G.; Lazdins, J.; Aboul-ela, F.; Varani, G.; Karn, J.; Klimkait, T. Proc. Natl. Acad. Sci. U. S. A. 1997, 94, 3548-3553. doi:10.1073/pnas.94.8.3548

7. Stawikowski, M.; Stawikowska, R.; Jaśkiewicz, A.; Zabłotna, E.; Rolka, K. ChemBioChem 2005, 6, 1057-1061. doi:10.1002/cbic.200400412

8. Panda, S. S.; El-Nachef, C.; Bajaj, K.; Katritzky, A. R. Eur. J. Org. Chem. 2013, 4156-4162. doi:10.1002/ejoc.201201731

9. Kessler, H. Angew. Chem., Int. Ed. Engl. 1993, 32, 543-544. doi:10.1002/anie.199305431

10. Yoo, B.; Kirshenbaum, K. Curr. Opin. Chem. Biol. 2008, 12, 714-721. doi:10.1016/j.cbpa.2008.08.015

11. Zuckermann, R. N. Pept. Sci. 2011, 96, 545-555. doi:10.1002/bip.21573

12. Simon, R. J.; Kania, R. S.; Zuckermann, R. N.; Huebner, V. D.; Jewell, D. A.; Banville, S.; Ng, S.; Wang, L.; Rosenberg, S.; Marlowe, C. K.; Spellmeyer, D. C.; Tan, R.; Frankel, A. D.; Santi, D. V.; Cohen, F. E.; Bartlett, P. A. Proc. Natl. Acad. Sci. U. S. A. 1992, 89, 9367-9371. doi:10.1073/pnas.89.20.9367

13. Udugamasooriya, D. G.; Dineen, S. P.; Brekken, R. A.; Kodadek, T. J. Am. Chem. Soc. 2008, 130, 5744-5752. doi:10.1021/ja711193x

14. Wrenn, S. J.; Weisinger, R. M.; Halpin, D. R.; Harbury, P. B. J. Am. Chem. Soc. 2007, 129, 13137-13143. doi:10.1021/ja073993a

15. Zuckermann, R. N.; Martin, E. J.; Spellmeyer, D. C.; Stauber, G. B.; Shoemaker, K. R.; Kerr, J. M.; Figliozzi, G. M.; Goff, D. A.; Siani, M. A.; Simon, R. J.; Banville, S. C.; Brown, E. G.; Wang, L.; Richter, L. S.; Moss, W. H. J. Med. Chem. 1994, 37, 2678-2685. doi:10.1021/jm00043a007
16. Rezai, T.; Bock, J. E.; Zhou, M. V.; Kalyanaraman, C.; Lokey, R. S.; Jacobson, M. P. J. Am. Chem. Soc. 2006, 128, 14073-14080. doi:10.1021/ja063076p

17. Ovadia, O.; Linde, Y.; Haskell-Luevano, C.; Dirain, M. L.; Sheynis, T.; Jelinek, R.; Gilon, C.; Hoffman, A. Bioorg. Med. Chem. 2010, 18, 580-589. doi:10.1016/j.bmc.2009.12.010

18. Gangloff, N.; Ulbricht, J.; Lorson, T.; Schlaad, H.; Luxenhofer, R. Chem. Rev. 2016, 116, 1753-1802. doi:10.1021/acs.chemrev.5b00201

19. Sun, J.; Zuckermann, R. N. ACS Nano 2013, 7, 4715-4732. doi:10.1021/nn4015714

20. Sarma, B. K.; Kodadek, T. ACS Comb. Sci. 2012, 14, 558-564. doi:10.1021/co3000852

21. Proulx, C.; Sabatino, D.; Hopewell, R.; Spiegel, J.; Ramos, Y. G.; Lubell, W. D. Future Med. Chem. 2011, 3, 1139-1164. doi:10.4155/fmc. 11.74

22. Zega, A. Curr. Med. Chem. 2005, 12, 589-597. doi: $10.2174 / 0929867053362802$

23. Gante, J. Synthesis 1989, 405-413. doi:10.1055/s-1989-27269

24. Gante, J. Angew. Chem., Int. Ed. Engl. 1970, 9, 813. doi:10.1002/anie.197008131

25. Bailey, M. D.; Halmos, T.; Goudreau, N.; Lescop, E.; Llinàs-Brunet, M. J. Med. Chem. 2004, 47, 3788-3799. doi:10.1021/jm049864b

26. Randolph, J. T.; Zhang, X.; Huang, P. P.; Klein, L. L.; Kurtz, K. A.; Konstantinidis, A. K.; He, W.; Kati, W. M.; Kempf, D. J. Bioorg. Med. Chem. Lett. 2008, 18, 2745-2750. doi:10.1016/j.bmcl.2008.02.053

27. Magrath, J.; Abeles, R. H. J. Med. Chem. 1992, 35, 4279-4283. doi:10.1021/jm00101a004

28. Baggio, R.; Shi, Y.-Q.; Wu, Y.-q.; Abeles, R. H. Biochemistry 1996, 35, 3351-3353. doi:10.1021/bi952879e

29. Xing, R.; Hanzlik, R. P. J. Med. Chem. 1998, 41, 1344-1351. doi: $10.1021 / \mathrm{jm} 970802 \mathrm{~d}$

30. Ekici, Ö. D.; Li, Z. Z.; Campbell, A. J.; James, K. E.; Asgian, J. L.; Mikolajczyk, J.; Salvesen, G. S.; Ganesan, R.; Jelakovic, S.; Grütter, M. G.; Powers, J. C. J. Med. Chem. 2006, 49, 5728-5749. doi:10.1021/jm0601405

31. Guy, L.; Vidal, J.; Collet, A.; Amour, A.; Reboud-Ravaux, M. J. Med. Chem. 1998, 41, 4833-4843. doi:10.1021/jm980419o

32. Amour, A.; Collet, A.; Dubar, C.; Reboud-Ravaux, M. Int. J. Pept. Protein Res. 1994, 43, 297-304. doi:10.1111/j.1399-3011.1994.tb00394.x

33. Bushkova, E.; Parchinsky, V.; Krasavin, M. Mol. Diversity 2010, 14, 493-499. doi:10.1007/s11030-009-9200-6

34. Krasavin, M.; Bushkova, E.; Parchinsky, V.; Shumsky, A. Synthesis 2010, 933-942. doi:10.1055/s-0029-1219274

35. Lakontseva, E.; Krasavin, M. Tetrahedron Lett. 2010, 51, 4095-4099. doi:10.1016/j.tetlet.2010.05.133

36. Bonnet, D.; Samson, F.; Rommens, C.; Gras-masse, H.; Melnyk, O. J. Pept. Res. 1999, 54, 270-278. doi:10.1034/j.1399-3011.1999.00105.x

37. Bordessa, A.; Keita, M.; Maréchal, X.; Formicola, L.; Lagarde, N.; Rodrigo, J.; Bernadat, G.; Bauvais, C.; Soulier, J.-L.; Dufau, L.; Milcent, T.; Crousse, B.; Reboud-Ravaux, M.; Ongeri, S. Eur. J. Med. Chem. 2013, 70, 505-524. doi:10.1016/j.ejmech.2013.09.059

38. Laurencin, M.; Legrand, B.; Duval, E.; Henry, J.; Baudy-Floc'h, M.; Zatylny-Gaudin, C.; Bondon, A. J. Med. Chem. 2012, 55, 2025-2034. doi:10.1021/jm2011595

39. Niedrich, H.; Köller, G. J. Prakt. Chem. 1974, 316, 729-740. doi:10.1002/prac. 19743160504 
40. Sarma, B. K.; Liu, X.; Kodadek, T. Bioorg. Med. Chem. 2016, 24 , 3953-3963. doi:10.1016/j.bmc.2016.04.047

41. Cheguillaume, A.; Lehardy, F.; Bouget, K.; Baudy-Floc'h, M.; Le Grel, P. J. Org. Chem. 1999, 64, 2924-2927. doi:10.1021/jo981487I

42. Bouget, K.; Aubin, S.; Delcros, J.-G.; Arlot-Bonnemains, Y.; Baudy-Floc'h, M. Bioorg. Med. Chem. 2003, 11, 4881-4889. doi:10.1016/j.bmc.2003.09.018

43. Aubin, S.; Martin, B.; Delcros, J.-G.; Arlot-Bonnemains, Y.; Baudy-Floc'h, M. J. Med. Chem. 2005, 48, 330-334. doi:10.1021/jm049455f

44. Lee, J.; Huang, W.; Broering, J. M.; Barron, A. E.; Seo, J. Bioorg. Med. Chem. Lett. 2015, 25, 2849-2852. doi:10.1016/j.bmcl.2015.04.092

45. Østergaard, S.; Holm, A. Mol. Diversity 1997, 3, 17-27. doi:10.1023/A:1009698507588

46. Zuckermann, R. N.; Kerr, J. M.; Kent, S. B. H.; Moos, W. H. J. Am. Chem. Soc. 1992, 114, 10646-10647. doi:10.1021/ja00052a076

47. Sarma, B. K.; Yousufuddin, M.; Kodadek, T. Chem. Commun. 2011, 47, 10590-10592. doi:10.1039/c1cc12750k

48. Fara, M. A.; Diaz-Mochón, J. J.; Bradley, M. Tetrahedron Lett. 2006, 47, 1011-1014. doi:10.1016/j.tetlet.2005.11.127

49. Dömling, A.; Ugi, I. Angew. Chem., Int. Ed. 2000, 39, 3168-3210. doi:10.1002/1521-3773(20000915)39:18<3168::AID-ANIE3168>3.0.CO ;2-U

50. Wessjohann, L. A.; Rivera, D. G.; Vercillo, O. E. Chem. Rev. 2009, 109, 796-814. doi:10.1021/cr8003407

51. Dömling, A. Chem. Rev. 2006, 106, 17-89. doi:10.1021/cr0505728

52. Dömling, A.; Beck, B.; Eichelberger, U.; Sakamuri, S.; Menon, S.; Chen, Q.-Z.; Lu, Y.; Wessjohann, L. A. Angew. Chem., Int. Ed. 2006, 45, 7235-7239. doi:10.1002/anie.200601259

53. Barreto, A. F. S.; Vercillo, O. E.; Birkett, M. A.; Caulfied, J. C.; Wessjohann, L. A.; Andrade, C. K. Z. Org. Biomol. Chem. 2011, 9, 5024-5027. doi:10.1039/c1ob05471f

54. Pando, O.; Stark, S.; Denkert, A.; Porzel, A.; Preusentanz, R.; Wessjohann, L. A. J. Am. Chem. Soc. 2011, 133, 7692-7695. doi:10.1021/ja2022027

55. Neves Filho, R. A. W.; Westermann, B.; Wessjohann, L. A. Beilstein J. Org. Chem. 2011, 7, 1504-1507. doi:10.3762/bjoc.7.175

56. Vercillo, O. E.; Andrade, C. K. Z.; Wessjohann, L. A. Org. Lett. 2008, 10, 205-208. doi:10.1021/ol702521g

57. Barreto, A. F. S.; Vercillo, O. E.; Wessjohann, L. A.; Andrade, C. K. Z. Beilstein J. Org. Chem. 2014, 10, 1017-1022. doi:10.3762/bjoc.10.101

58. Constabel, F.; Ugi, I. Tetrahedron 2001, 57, 5785-5789. doi:10.1016/S0040-4020(01)00516-6

59. Dömling, A. Nucleosides Nucleotides 1998, 17, 1667-1670. doi:10.1080/07328319808004699

60. Xu, P.; Zhang, T.; Wang, W.; Zou, X.; Zhang, X.; Fu, Y. Synthesis 2003, 1171-1176. doi:10.1055/s-2003-39391

61. Zarganes-Tzitzikas, T.; Patil, P.; Khoury, K.; Herdtweck, E.; Domling, A. Eur. J. Org. Chem. 2015, 51-55. doi:10.1002/ejoc.201403401

62. Brauch, S.; Gabriel, L.; Westermann, B. Chem. Commun. 2010, 46, 3387-3389. doi:10.1039/b927388c

63. Yale, H. L.; Losee, K.; Martins, J.; Holsing, M.; Perry, F. M.; Bernstein, J. J. Am. Chem. Soc. 1953, 75, 1933-1942. doi:10.1021/ja01104a046

64. Bruice, T. C.; Benkovic, S. J. J. Am. Chem. Soc. 1964, 86, 418-426. doi:10.1021/ja01057a026

\section{License and Terms}

This is an Open Access article under the terms of the Creative Commons Attribution License

(http://creativecommons.org/licenses/by/4.0), which permits unrestricted use, distribution, and reproduction in any medium, provided the original work is properly cited.

The license is subject to the Beilstein Journal of Organic Chemistry terms and conditions:

(http://www.beilstein-journals.org/bjoc)

The definitive version of this article is the electronic one which can be found at:

doi:10.3762/bjoc. 12.285 